\title{
Coherent Eavesdropping Attacks in Quantum Cryptography: Nonequivalence of Quantum and Classical Key Distillation
}

\author{
Dagomir Kaszlikowski, ${ }^{1}$ Jenn Yang Lim, ${ }^{1}$ Leong Chuang Kwek, ${ }^{2,1}$ and Berthold-Georg Englert ${ }^{1}$ \\ ${ }^{1}$ Department of Physics, National University of Singapore, Singapore 117542, Singapore \\ ${ }^{2}$ National Institute of Education, Nanyang Technological University, Singapore 639798, Singapore
}

(Dated: 20 December 2003)

\begin{abstract}
The security of a cryptographic key that is generated by communication through a noisy quantum channel relies on the ability to distill a shorter secure key sequence from a longer insecure one. We show that - for protocols that use quantum channels of any dimension and completely characterize them by state tomography - the noise threshold for classical advantage distillation is substantially lower than the threshold for quantum entanglement distillation because the eavesdropper can perform powerful coherent attacks. The earlier claims that the two noise thresholds are identical, which were based on analyzing incoherent attacks only, are therefore invalid.

PACS numbers: 03.67.Dd, 03.65.Wj, 03.67.Hk
\end{abstract}

Unavoidably, all practical implementations of protocols for quantum cryptography suffer from noise in the quantum channel and must, therefore, face the problem of generating a secure cryptographic key from noisy raw data. Since the noise can wholly result from eavesdropping attacks, the central question is: Where is the noise threshold below which the communicating parties, Alice and Bob, can generate a secure key at all?

Except at rather low noise levels, the extraction of a shorter secure key sequence from a longer, noisy and insecure, raw key sequence must involve a distillation procedure, either a variant of "quantum entanglement distillation" (QED 1]) or of "classical advantage distillation" (CAD 2 2 ). A recent paper asserts that, for an important class of protocols, "the thresholds for QED and CAD are the same" so that "the two distillation procedures are equivalent in the sense that neither offers a security advantage over the other" [3] , and the same conclusion has also been reached in Ref. [4], and earlier in Ref. 5] for qubit protocols.

But the analysis in Refs. [3, 4, 5] takes only incoherent attacks into account, in which eavesdropper Eve acquires as much information as possible about each individual entry in the raw key data shared by Alice and Bob. This raw key is, however, of secondary interest to Eve, who primarily wishes to know well the final, distilled key. Accordingly, she might be better off by satisfying her primary interest directly, rather than first maximizing her transitory knowledge of secondary interest.

Indeed, we report here that Eve can gain more knowledge about the final key sequence by a suitable coherent attack, if Alice and Bob make use of CAD. The actual noise threshold for CAD is therefore lower than the one found in Refs. $3,4,5]$. And since the threshold for QED is independent of Eve's eavesdropping strategy, it follows that the two distillation procedures are not equivalent: The noise level in a quantum channel can be above the CAD threshold but below the QED threshold.

In this context, then, coherent eavesdropping attacks truly outperform incoherent attacks. As plausible as this may seem in hindsight, it is not at all obvious. In fact, when neither QED nor CAD are performed, it has been argued that coherent attacks cannot be more powerful than incoherent attacks [ 6 ].

We reconsider the tomographic protocol for quantum cryptography of Refs. 7] and [3]. A source distributes pairs of qunits ( $n$-dimensional quantum objects, $n \geq 2$ ) to Alice and Bob, and they measure nondegenerate observables that are randomly chosen from a tomographically complete set of $n+1$ observables. Both keep a record of the observable they have measured for each pair and of the measurement result. As in Refs. 3, 7] we denote by $\left|m_{k}\right\rangle$ the $k$ th eigenket of Alice's $m$ th observable and by $\left|\bar{m}_{k}\right\rangle$ the $k$ th eigenket of Bob's $m$ th observable. The correspondence between the orthonormal bases associated with the observables is established by requiring that

$$
\left\langle 0_{j} \mid m_{k}\right\rangle=\left\langle\bar{m}_{k} \mid \overline{0}_{j}\right\rangle
$$

holds for $j, k=0,1,2, \ldots, n-1$ and $m=0,1,2, \ldots, n$. We are thus pairing Alice's $m$ th observable with Bob's $m$ th observable.

This pairing is essential in defining the two-qunit state

$$
|\psi\rangle=\frac{1}{\sqrt{n}} \sum_{k=0}^{n-1}\left|m_{k} \bar{m}_{k}\right\rangle
$$

that Alice and Bob wish to receive from an ideal source. The two-qunit kets $\left|m_{k} \bar{m}_{k}\right\rangle$ refer to the $m$ th pair of observables but, as a consequence of Eq. (10), $|\psi\rangle$ is the same regardless of the $m$ value chosen in (2).

When the transmission is over, Alice and Bob publicly announce their choice of observables, their respective $m$ values, for all qunits, while keeping the measurement results, their nit values, secret. The qunit pairs constitute two groups, one in which the measurement bases match (both $m$ values are the same, which happens with probability $1 /(n+1)$ ), and the other in which the bases do 
not match. In the absence of noise, the nit values of the first group are perfectly correlated and thus give rise to a cryptographic key in an alphabet with $n$ letters.

In reality, however, Alice and Bob must take into account Eve's attempts at eavesdropping and the resulting disturbance of the quantum channel. As a consequence, the source effectively emits qunit pairs whose properties are described by a statistical operator $\rho$ that differs from the ideal projector $|\psi\rangle\langle\psi|$. Since Alice and Bob measure tomographically complete sets of observables on their respective qunits, they can determine the actual $\rho$ from their measurement results. They exploit all data of the mismatched bases for this purpose, and some of the matched-bases data. Ideally, they wish for $\rho=|\psi\rangle\langle\psi|$ but, realistically, they expect to find a $\rho$ of the form

$$
\rho=|\psi\rangle\left(\beta_{0}-\beta_{1}\right)\left\langle\psi\left|+\frac{\beta_{1}}{n}=\right| \psi\right\rangle(1-\mathcal{E})\langle\psi|+\frac{\mathcal{E}}{n^{2}},
$$

with $\beta_{0}+(n-1) \beta_{1}=1$ and $\mathcal{E}=1-\left(\beta_{0}-\beta_{1}\right)=n \beta_{1}$. This is what one gets when an imperfect transmission line admixes unbiased noise to $|\psi\rangle\langle\psi|$, the fraction of the admixture being quantified by the noise parameter $\mathcal{E}[8]$. The nonnegative parameters $\beta_{0}$ and $\beta_{1}$ have the following physical significance: $\beta_{0}$ is the probability that Alice and Bob get the same nit value when the bases match, and $\beta_{1}$ is the probability that Bob gets a particular one of the $n-1$ values that are different from Alice's nit value.

The relevant range of parameters is such that $0 \leq \beta_{1} \leq$ $\beta_{0} \leq 1$ or $0 \leq \mathcal{E} \leq 1$ as only then the state $\rho$ has the interpretation of an admixture of noise to the pure state $|\psi\rangle$. The limiting values mark the extreme situations of "no noise at all" $\left(\beta_{0}=1, \beta_{1}=0, \mathcal{E}=0\right)$ and "nothing but noise" $\left(\beta_{0}=\beta_{1}=1 / n, \mathcal{E}=1\right)$.

Sources that emit two-qunit states $\rho$ of a kind different from the one in Eq. (3) are not regarded as trustworthy by Alice and Bob. As the crucial, defining step of the tomographic protocol, they accept the source only if their state tomography confirms that the source emits statistically independent qunit pairs with a $\rho$ of the form (3). Otherwise, they switch to another source [9].

We grant Eve full control over the two-qunit source. Then, in order to acquire as much information about the key as possible, she entangles one ancilla each with the qunit pairs sent to Alice and Bob. Since they must receive the pairs in the state (3), Eve's choices are severely limited. She is bound to prepare entangled two-qunitancilla states with a ket of the form [7]

$$
\begin{aligned}
|\Psi\rangle= & \sqrt{\frac{\beta_{0}}{n}} \sum_{k=0}^{n-1}\left|m_{k} \bar{m}_{k}\right\rangle\left|E_{k k}^{(m)}\right\rangle \\
& +\sqrt{\frac{\beta_{1}}{n}} \sum_{k \neq l}\left|m_{k} \bar{m}_{l}\right\rangle\left|E_{k l}^{(m)}\right\rangle,
\end{aligned}
$$

where the $\left|E_{k l}^{(m)}\right\rangle$ are normalized ancilla kets. The sets of ancilla states pertaining to different values of $m$ are unitarily equivalent. For a given $m$ value, the ancilla states $\left|E_{k l}^{(m)}\right\rangle$ with $k \neq l$ are orthogonal to each other and orthogonal to the ones with $k=l$. The latter are not orthogonal among themselves (except when $\beta_{0}=\beta_{1}$, the case of pure noise and of very little interest), but rather have the same inner products for all pairs, i.e.,

$$
\left\langle E_{k k}^{(m)} \mid E_{l l}^{(m)}\right\rangle=1-\frac{\beta_{1}}{\beta_{0}}=\frac{1-\mathcal{E}}{1-(1-1 / n) \mathcal{E}} \equiv \lambda
$$

for $k \neq l$.

Alice and Bob can generate a secure key if the correlations between their nit values (i.e. their measurement results for matched bases) are stronger than the correlations between, say, Alice's values and the values that Eve obtains by whatever measurements on the respective ancillas. In technical terms, the mutual information between Alice and Bob must be larger than the mutual information between Alice and Eve 10. The efficiency of the protocol is proportional to the difference between the two mutual information values.

If $\mathcal{E}$ is sufficiently small, this condition is already met for the raw key sequence (see Ref. 11 for the actual criterion), and then the generation of the secure key is a matter of applied coding theory. Alice and Bob know if this is the case because they have determined the actual value of $\mathcal{E}$ by the two-qunit-state tomography.

If $\mathcal{E}$ is found to be not "sufficiently small," then the raw data is too noisy and Alice and Bob must use a distillation procedure to improve the situation, either QED or CAD; see Refs. 1] and [2], respectively. When employing QED, Alice and Bob process the qunit pairs before measuring the observables that give the nit values and so form, in essence, a purified set of qunit pairs with a new $\mathcal{E}$ value that is small enough. As established in Ref. [1], QED can be performed successfully if $\beta_{0}>2 \beta_{1}$ and only then. In terms of the noise parameter $\mathcal{E}$ this means

$$
\mathcal{E}<\mathcal{E}_{\text {th }}^{(\mathrm{QED})}=\frac{n}{n+1},
$$

which thus identifies the noise threshold for QED. The two-qunit state of (3) is separable if $\mathcal{E} \geq \mathcal{E}_{\text {th }}^{(\mathrm{QED})}$, so that QED exhausts the full range of $\mathcal{E}$ values for which cryptographic security is potentially possible, and no other procedure can ever have a larger $\mathcal{E}$ range.

Whereas the qunits are manipulated in QED, one processes the measured nit values when performing CAD. Therefore, the implementation of QED is a very challenging hardware problem whereas rather simple software is needed for CAD. This practical advantage of CAD over QED comes, however, at a price. As we now proceed to demonstrate, the noise threshold for CAD is lower than the QED threshold of Eq. (6).

In the CAD protocol Alice and Bob divide their raw key sequence of nit values (for the matched bases) into blocks of length $L$. For each block Alice tosses an $n$-sided 
die and adds, modulo $n$, the resulting random value to each value of the block. She so obtains a new block, which she sends to Bob through an authenticated but insecure public channel. After receiving the block, he subtracts his corresponding block from it (modulo $n$ ). If all the nit values are the same after the subtraction, which happens with probability $\beta_{0}^{L}+(n-1) \beta_{1}^{L}$, Bob informs Alice that this is a good block, otherwise it is a bad block [9].

All good blocks together define a distilled sequence of nit values, one value for each block: Alice records the random nit values she added, Bob the nit values he found after the subtraction. The distilled sequence can be characterized by probabilities $\beta_{0}^{(L)}$ (Bob has the same value as Alice) and $\beta_{1}^{(L)}$ (he has a particular one of the $n-1$ other values) that are the $L>1$ analogs of their $L=1$ versions in Eq. (3) and related to them by [3]

$$
\frac{\beta_{1}^{(L)}}{\beta_{0}^{(L)}}=\left(\frac{\beta_{1}}{\beta_{0}}\right)^{L} \text {. }
$$

Accordingly, with growing $L$, the distilled sequence has exponentially less noise than the raw sequence.

After performing CAD, the resulting mutual information between Alice and Bob is given by

$$
I_{L}(A \& B)=1+\beta_{0}^{(L)} \log _{n} \beta_{0}^{(L)}+\left(1-\beta_{0}^{(L)}\right) \log _{n} \beta_{1}^{(L)}
$$

with

$$
\left(\beta_{0}^{(L)}, \beta_{1}^{(L)}\right)=\frac{\left(\beta_{0}^{L}, \beta_{1}^{L}\right)}{\beta_{0}^{L}+(n-1) \beta_{1}^{L}},
$$

where, for convenience, we measure information in nits $\left(\log _{n}\right)$ rather than bits $\left(\log _{2}\right)$. The asymptotic forms

$$
\begin{aligned}
& \beta_{0}^{(L)} \simeq 1-(n-1)\left(\beta_{1} / \beta_{0}\right)^{L}, \quad \beta_{1}^{(L)} \simeq\left(\beta_{1} / \beta_{0}\right)^{L}, \\
& I_{L}(A \& B) \simeq 1-(n-1)\left(\beta_{1} / \beta_{0}\right)^{L} \log _{n}\left(\beta_{0} / \beta_{1}\right)^{L}
\end{aligned}
$$

apply for $L \gg 1$, so that the difference $1-I_{L}(A \& B)$ decreases exponentially with increasing block length $L$.

Eve's strategy is as follows. She stores her ancillas and waits passively until Bob announces his approval or rejection of the given block to Alice over the public channel. The ancillas of the bad blocks are then of no further interest. For each good block Eve knows that either (I) all corresponding nit values in Alice's and Bob's blocks are the same, or (II) they differ by the same amount (modulo $n$ ). There is no room for any other possibility in the CAD protocol. For instance, Alice could have the block 0121 for $L=4$ and $n=3$, and then there are three possible blocks for Bob, namely 0121, 1202, or 2010, resulting from Alice's addition of 0,1 , or 2 , respectively. The fraction $\beta_{0}^{(L)}$ of the good blocks are case-I blocks, the fraction $(n-1) \beta_{1}^{(L)}=1-\beta_{0}^{(L)}$ are case-II blocks.

For each good block, Eve has a corresponding set of ancilla states. Rather than measuring the ancillas oneby-one (incoherent attack), she performs a joint measurement on all $L$ of them (coherent attack) to acquire knowledge about the value that, say, Alice assigns to the block.
In case (II), Eve knows exactly Alice's and Bob's nit values because all the respective ancilla states $\left|E_{k l}^{(m)}\right\rangle$ have $k \neq l$ and are thus orthogonal to all other potential ones (recall the remark after Eq. (4)) and can be distinguished unambiguously.

In case (I), Alice and Bob have an identical block of nit values to begin with, and Eve's ancillas states are all of the $k=l$ kind. Although she can establish easily that the blocks are of case (I), she cannot distinguish the potential ancilla states unambiguously because they are not orthogonal to each other. Accordingly, Eve has no certain knowledge of the distilled nit values for case-I blocks. But by making good use of the classical information that is exchanged publicly between Alice and Bob during the distillation process, Eve can learn a lot about these nit values. In fact, she only needs to distinguish $n$ possible $L$-ancillas states, and they are almost orthogonal to each other when $L \gg 1$.

The situation is best illustrated with an example. Suppose and Alice and Bob have the same block 0121 for $n=3$ and $L=4$, and her random nit value is 1 . After addition (modulo 3), she sends the processed block 1202 to Bob via a public channel. Eve, who is fully knowledgeable of all such broadcast information and has already established that she is dealing with a case-I block, then infers that the unprocessed block is either 1202 , or 0121, or 2010 , and the distilled values would be 0,1 , and 2 , respectively. She concludes that the four ancillas in question are in the four-ancilla state with ket $\left|E_{11} E_{22} E_{00} E_{22}\right\rangle$, or $\left|E_{00} E_{11} E_{22} E_{11}\right\rangle$, or $\left|E_{22} E_{00} E_{11} E_{00}\right\rangle$, where the $E_{k k}^{(m)}$, s at the same positions have identical $m$ values that we leave implicit. Any two of these four-ancilla states have the same inner product of $\lambda^{4}$, inasmuch as

$$
\begin{aligned}
& \left\langle E_{11} E_{22} E_{00} E_{22} \mid E_{00} E_{11} E_{22} E_{11}\right\rangle \\
= & \left\langle E_{11} \mid E_{00}\right\rangle\left\langle E_{22} \mid E_{11}\right\rangle\left\langle E_{00} \mid E_{22}\right\rangle\left\langle E_{22} \mid E_{11}\right\rangle=\lambda^{4},
\end{aligned}
$$

for instance. More generally, for each case-I block of length $L$, Eve needs to distinguish $n$ possible $L$-ancilla states, with inner products of $\lambda^{L}$ for each pair of states.

For large $L$, this inner product is very small and, therefore 11], Eve maximizes her mutual information with Alice (or Bob) by the so-called "square-root measurement", which is always the error-minimizing measurement. Her probability of inferring a distilled case-I nit value correctly is then given by [7]

$$
\eta_{0}^{(L)}=\left(\frac{\sqrt{1+(n-1) \lambda^{L}}+(n-1) \sqrt{1-\lambda^{L}}}{n}\right)^{2},
$$

and she gets a particular one of the $n-1$ wrong values with probability

$$
\eta_{1}^{(L)}=\frac{1-\eta_{0}^{(L)}}{n-1}=\left(\frac{\sqrt{1+(n-1) \lambda^{L}}-\sqrt{1-\lambda^{L}}}{n}\right)^{2} .
$$


The resulting mutual information between Alice and Eve is

$$
\begin{aligned}
I_{L}(A \& E)= & 1+\beta_{0}^{(L)}\left(\eta_{0}^{(L)} \log _{n} \eta_{0}^{(L)}\right. \\
& \left.+\left(1-\eta_{0}^{(L)}\right) \log _{n} \eta_{1}^{(L)}\right) .
\end{aligned}
$$

The asymptotic forms

$$
\begin{aligned}
& \eta_{0}^{(L)} \simeq 1-\frac{1}{4}(n-1) \lambda^{2 L}, \quad \eta_{1}^{(L)} \simeq \frac{1}{4} \lambda^{2 L}, \\
& I_{L}(A \& E) \simeq 1-\frac{1}{4}(n-1) \lambda^{2 L} \log _{n}(1 / \lambda)^{2 L}
\end{aligned}
$$

apply for $L \gg 1$, so that the difference $1-I_{L}(A \& E)$ also decreases exponentially with increasing block length $L$.

Now, according to the Csiszár-Körner Theorem [10], a secure cryptographic key can be generated from the raw key sequence, by means of a suitably chosen errorcorrecting code and classical (one-way) communication between Alice and Bob, if the mutual information between Alice and Bob exceeds that between Eve and either of them. This ensures success of the CAD procedure whenever $I_{L}(A \& B)>I_{L}(A \& E)$ obtains.

Upon comparing the large- $L$ versions of the mutual informations in (10) and (14), we note that, for sufficiently long blocks, successful CAD is assuredly possible if $\beta_{1} / \beta_{0}<\lambda^{2}$. Since $\lambda+\beta_{1} / \beta_{0}=1$, the corresponding criterion for the noise level is

$$
\mathcal{E}<\mathcal{E}_{\text {th }}^{(\mathrm{CAD})}=\frac{n}{n+(1+\sqrt{5}) / 2} .
$$

The CAD threshold value thus identified is always lower than the QED threshold value of Eq. (6), because the golden mean $(1+\sqrt{5}) / 2$ exceeds unity.

Figure 1 shows the two noise thresholds as a function of $n$, for $2 \leq n \leq 30$. It can be seen clearly that QED can tolerate substantially more noise in the channel than $\mathrm{CAD}$, in particular in the qubit case of $n=2$, where the thresholds are at $\mathcal{E}=2 / 3=66.7 \%$ and $\mathcal{E}=1-\sqrt{1 / 5}=55.3 \%$, respectively.

In summary, we have established that Eve has a real advantage from coherent attacks if Alice and Bob perform CAD. The coherent attack that we describe in detail aims at getting optimal knowledge about each nit value of the distilled key individually. It is conceivable (but we do not consider it likely) that more involved coherent attacks, which would provide knowledge about groups of distilled-key nit values, are even more powerful. Strictly speaking, the threshold stated in Eq. (15) must, therefore, be regarded as an upper bound on the noise threshold for CAD.

We note further that other procedures for advantage distillation are also vulnerable to coherent attacks of the considered kind. This is true, in particular, for the paritycheck distillation for qubit protocols, for which an analogous coherent attack can be analyzed easily [12].

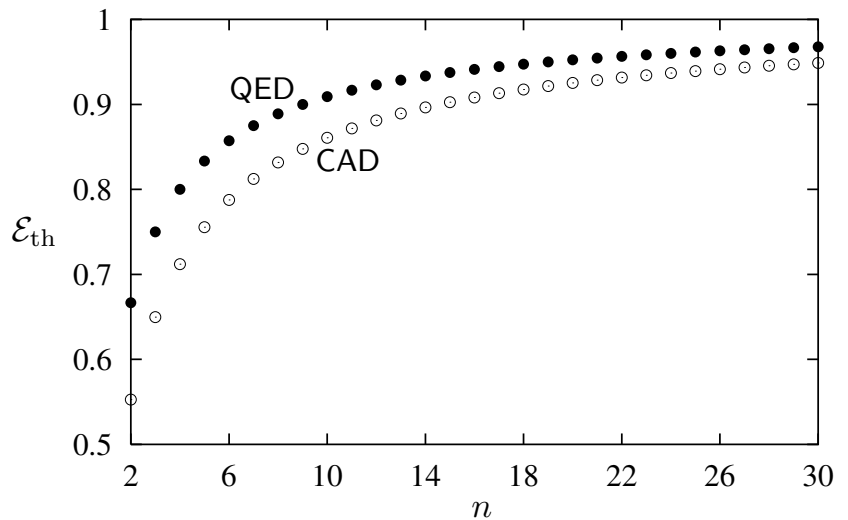

FIG. 1: Noise thresholds $\mathcal{E}_{\text {th }}$ for Quantum Entanglement Distillation (QED, full circles) and Classical Advantage Distillation (CAD, empty circles) under coherent eavesdropping attacks, as a function of the dimension $n$ of the transmitted quantum objects. QED can tolerate substantially more noise in the channel.

We are indebted to Artur Ekert, Ajay Gopinathan, Christian Kurtsiefer, and Yeong Cherng Liang for sharing their insights with us. This work was supported by A*Star Grant No. 012-104-0040.

[1] M. Horodecki and P. Horodecki, Phys. Rev. A 59, 4206 (1999). We note that, in the context of quantum cryptography, QED was originally proposed for qubits by D. Deutsch, A. Ekert, R. Jozsa, C. Macchiavello, S. Popescu, and A. Sanpera, Phys. Rev. Lett. 77, 2818 (1996), under the name of Quantum Privacy Amplification.

[2] U. M. Maurer, IEEE Trans. Inf. Theory 39, 733 (1993).

[3] D. Bruß, M. Christandl, A. Ekert, B.-G. Englert, D. Kaszlikowski, and C. Macchiavello, Phys. Rev. Lett. 91, 097901 (2003).

[4] A. Acin, N. Gisin, and V. Scarani, eprint quant-ph/ 0303009 .

[5] N. Gisin and S. Wolf, Phys. Rev. Lett. 83, 4200 (1999).

[6] J. I. Cirac and N. Gisin, Phys. Lett. A229, 1 (1997); Wang Xiang-bin, eprint quant-ph/0110089.

[7] Y. C. Liang, D. Kaszlikowski, B.-G. Englert, L. C. Kwek, and C. H. Oh, Phys. Rev. A 68, 022324 (2003).

[8] The fidelity of the corresponding effective quantum channel between Alice and Bob is $\mathcal{F}=1-\left(1-1 / n^{2}\right) \mathcal{E}$.

[9] Eve is, of course, aware of this because, as required by the Kerkhoff Principle of cryptology, she knows all procedural rules of the protocol executed by Alice and Bob and has access to all information they exchange publicly.

[10] Csiszár and J. Körner, IEEE Trans. Inf. Theory 24, 339 (1978).

[11] D. Kaszlikowski, A. Gopinathan, Y. -C. Liang, L. C. Kwek, and B.-G. Englert, eprints quant-ph/0307086 and quant-ph/0310144

[12] D. Kaszlikowski, B.-G. Englert, and K. T. Cha (unpublished). 